\title{
Structural Characterization of Ancient Japanese Swords from MAAS Using Neutron Strain Scanning Measurements
}

\author{
Filomena Salvemini ${ }^{1, a^{*}}$, Vladimir Luzin ${ }^{1, b}$, Francesco Grazzi $^{2, c}$, Sue Gatenby ${ }^{3, d}$, \\ Min-Jung Kim ${ }^{3, \mathrm{e}}$ \\ ${ }^{1}$ ANSTO, Bragg Institute, Lucas Heights, NSW, Australia \\ ${ }^{2}$ CNR, Istituto dei Sistemi Complessi, Sesto Fiorentino, Italy \\ ${ }^{3}$ Museum of Applied Arts and Sciences, Sydney, Australia \\ a*filomena.salvemini@ansto.gov.au, bvladimir.luzin@ansto.gov.au, \\ 'francesco.grazzi@fi.isc.cnr.it, dsue.gatenby@maas.museum, ${ }^{d}$ min-jung.kim@maas.museum
}

\begin{abstract}
Keywords: Neutron Residual Stress, Non-Invasive Analysis, Archaeometallurgy, Japanese Sword
\end{abstract}

\begin{abstract}
The current paper presents a forensic study that aims to characterize non-invasively the laminated structure of a set of Samurai's swords, part of the East Asian Collection of the Museum of Applied Arts and Sciences (MAAS) in Sydney, via strain scanning measurements. Neutron residual stress analyses were undertaken on samples of well-known origin, time period and authorship to create a reference database on the main lamination methods developed by Japanese swordsmiths. The benchmark data were cross-matched with results obtained from a mumei (no-signature) blade in order to attribute its manufacturing tradition based on qualitative and quantitative data rather than stylistic criteria. Maps of two stress components and $\mathrm{d}_{0}$-values in the transverse crosssection of each sword were determined as a result of the neutron diffraction experiment with submillimeter spatial resolution. Since these two material characteristics are induced by the manufacturing process, analysis and comparison of the results allows drawing conclusions about variability or similarity of the actual production techniques of the Japanese swords.
\end{abstract}

\section{Introduction}

Japanese swords are famed among all others all over the world as the most effective in terms of hardness, resilience and, last but not least, aesthetics [1]. Their forging technique was almost unique; steel lumps, obtained from the furnace, were strongly pre-treated to obtain a homogenous and purified multilayered sheet. Distinctive carbon steels, characterized by different hardness, were shaped and specifically used for different parts of the blade components to optimize their mechanical feature (Fig. 1) [1].

Since the Koto age, $10^{\text {th }}-17^{\text {th }}$ century $A D$, five different traditions developed distinctive construction techniques that evolved during the following historical periods [2]. However, the actual techniques that were used by the early sword-smiths were never documented and the necessary information was orally transmitted from the master to his most skilled pupils. In spite of the large amount of studies published on the subject, different manufacturing techniques are still not fully understood.

Until recently, only expendable samples were investigated by standard analytical techniques, which mainly require sample cutting or are based on surface analysis. Nowadays, neutron diffraction [4] and neutron imaging methods [5] have been demonstrated to be the most suitable tools to qualitatively and quantitatively characterize composition and micro-structural properties of metal artifacts in a non-destructive way, mandatory for well conserved museum collections. 
In the current study we attempted systematic research of the Japanese blades in order to investigate whether the laminated structure of a set of Samurai's swords can be determined in non-destructive manner by means of neutron tomography, neutron diffraction stress analysis or both. The research is based on part of the East Asian Collection of the Museum of Applied Arts and Sciences (MAAS) in Sydney. The Japanese sword collection contains both samples of well-known origin, time period and authorship (group 1) as well as a group of mumei (no-signature) blades (group 2). Group 1 allowed us to build up a reference database on the main lamination methods adopted by Japanese swordsmiths and therefore to be used as benchmark data that can be cross-matched with the results obtained from group 2 in the attempt of attributing the corresponding manufacturing tradition to the unknown blades.

Although a number of different blades were analyzed, in the current work we report only the neutron diffraction stress analysis on blades which were classified as katana, while the neutron tomographic data and results on wakizashi

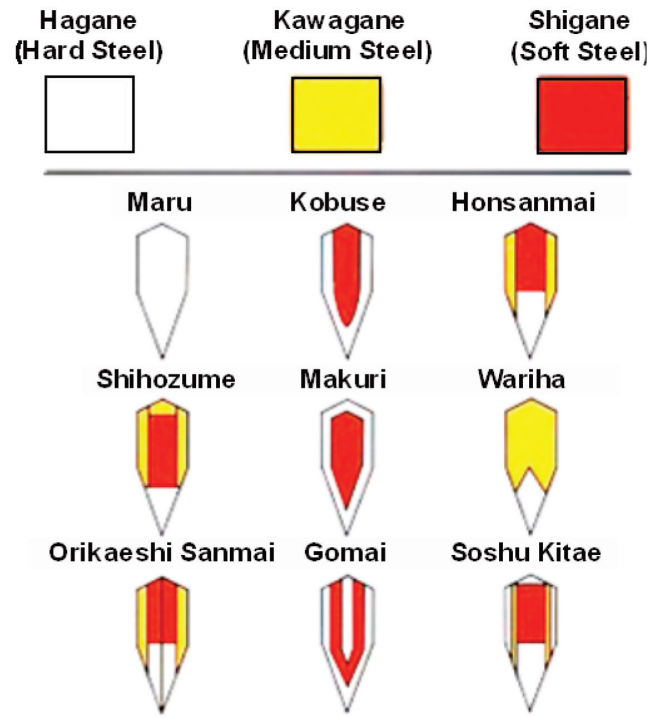

Fig. 1. The most common lamination structures of the Japanese swords. will be published separately somewhere else.

\section{Samples}

The attributes of the four katana from of the East Asian Collection of MAAS that were used in the forensic investigation are reported in Table. 1. Origin, time period and authorship are known only for three of them by transliteration of the signature engraved on their hilt. According to stylistic study, only the time period can be assigned for blade H6856 while the manufacturing tradition it belongs to is still uncertain.

The physical description of the swords is given in Table 2, where the blades' total length and thickness and width, measured in the mid length (where neutron diffraction measurement were made) are reported.

\section{Experimental: neutron diffraction stress analysis}

The neutron diffraction stress analysis in objects like Japanese swords is difficult because of two reasons. First, a technical aspect, a high spatial resolution is required, $\sim 0.5 \mathrm{~mm}$, since individual multiple layers can be of sub-millimeter size while a typical thickness of a blade is $5-7 \mathrm{~mm}$. At the same time many points are required to be measured since the exact number and location of layers are not quite known. Therefore a compromise between resolution, number of measurement points and experimental time per point needs to be found.

Second, a theoretical aspect, since the experimentally measured peak shift combines two effects, variation of (macrostress-free) $\mathrm{d} 0$ and elastic macrostress, and both of them are expected to be present due to manufacturing process, identifying and separation of these two contributions is essential for the aim of the experiment. However, there is no chance for proper resolution of $\mathrm{d} 0$ since only non-destructive analysis is allowed for the museum items. Thus,

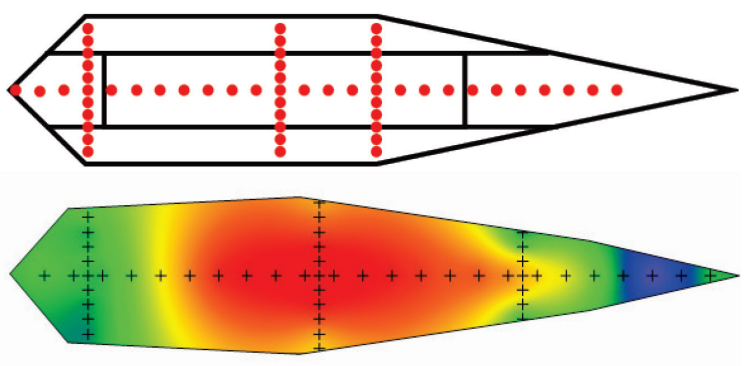

Fig. 2. A generic mesh in the transverse crosssection of the sword for neutron diffraction mapping and a real example of the longitudinal stress map for sword H4839 (min: -446 MPa, $\max : 291 \mathrm{MPa}$ ). 
the approach described in the following was used as the best possibility to resolve $\mathrm{d} 0$ problem.

The first problem was solved by use of ANSTO's neutron residual stress diffractometer KOWARI [6], which can provide necessary sub-millimeter $(0.5 \mathrm{~mm})$ spatial resolution and feasibility of that was proved in the past experiments [7]. Although a full 2D mapping would be very desirable, due to time limitations only a number of one-dimensional scans were performed. However, the measurement points are selected in such a manner and such numbers, $>50$, that would allow distinguishing unambiguously between possible structures as shown in Fig. 2 and at the same time some interpolation can be applied to produce 2D maps. At each mesh point spaced by $0.5 \mathrm{~mm}$ through thickness and by $1.0 \mathrm{~mm}$ along the central line, strain measurement was carried out in three orthogonal directions (longitudinal, transverse, normal) as in a traditional stress scanning experiment. A nominal gauge volume as small as $0.5 \times 0.5 \times 0.5 \mathrm{~mm} 3$ was used for measurements of the longitudinal strain component (symbols in Fig. 2 are scaled to this gauge volume size), while it was enlarged to $0.5 \times 0.5 \times 20 \mathrm{~mm} 3$ for measurement of the normal and transverse, since this was allowed by blade geometry, with extension of the gauge volume along the longitudinal direction. Strain measurement has been done using wavelength of $1.67 \AA$ that provides $\mathrm{Fe}(211)$ reflection at $2 \theta=90^{\circ}$ and typical accuracy of $\pm 30 \mu$ strain that was achieved for the normal and transverse components after an acquisition time of 4 minutes. For the longitudinal strain component, however, a typical accuracy was $\pm 50 \mu$ strain with a measurement time of 20 minutes. Overall, approximately 1.5 days of beamtime was used for each blade.

Table 2: Swords characterisation. Thickness and width are taken at the point of maximum curvature of the blade. The length is measured as a straight line from the point to the notch where the back of the katana meets the mounting of the hilt.

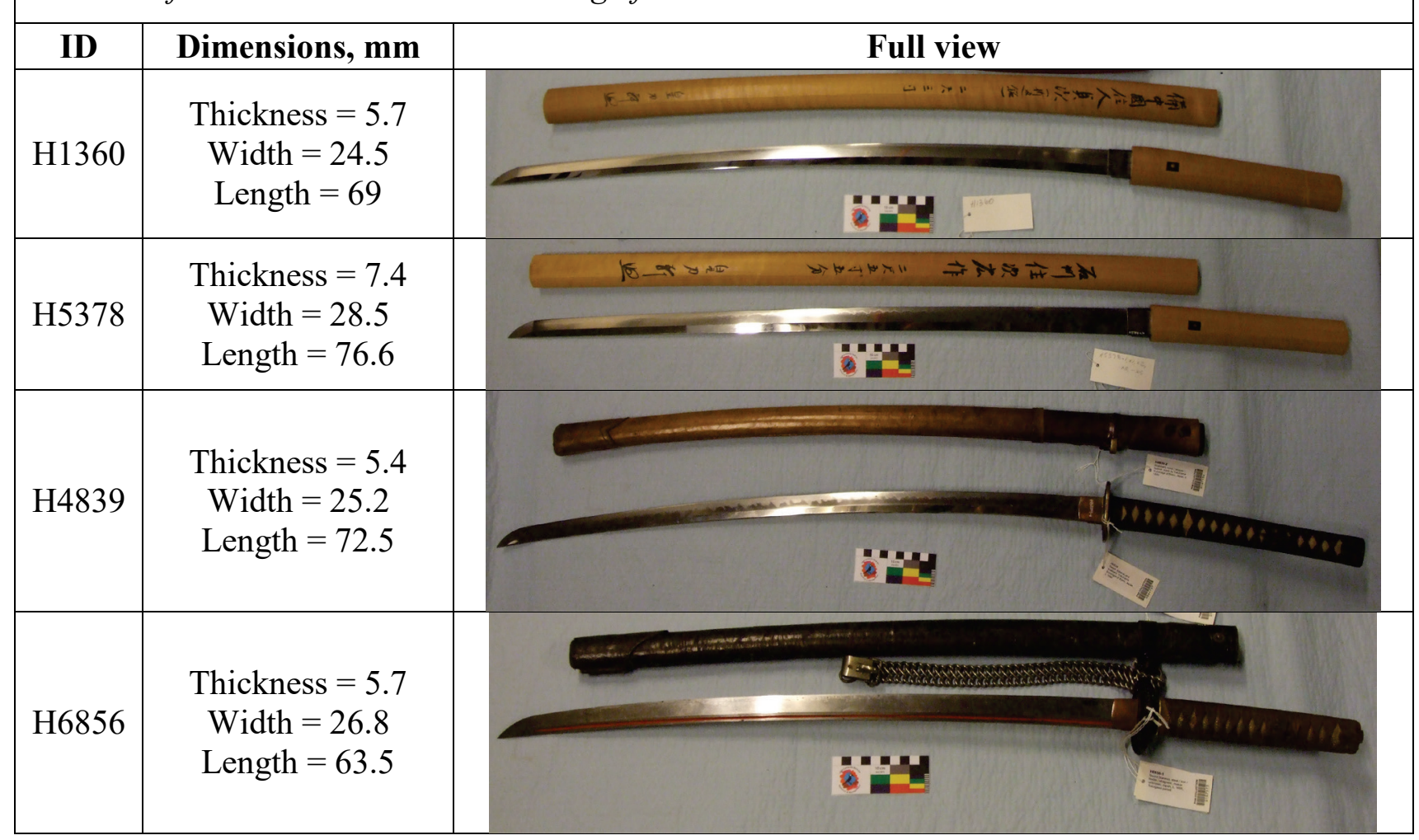

In order to resolve the second problem, the following strategy was adopted. In contrast to the traditional stress experiment, where $\mathrm{d}_{0}$ must be provided and three stress components are calculated, in the current experiment, the zero-through-thickness-stress condition was applied enabling calculation of two stress components and one $\mathrm{d}_{0}$-value out of the $\mathrm{d}$-spacings for three measured directions. This plane-stress condition should be fulfilled with good accuracy (within our 
experimental errors) because of sample planar geometry, but the major concern would be the anisotropic behaviour of $\mathrm{d}_{0}$. Until slicing of a blade is allowed, this is the only practical assumption. However, some checks can be applied to verify the assumption. If the abovementioned assumption is accurate enough and stress and $\mathrm{d}_{0}$ are resolved correctly then the transverse stress component should satisfy with boundary conditions on top and bottom edges of the blades and the longitudinal integral stress balance condition can be (at least approximately) evaluated.

\section{Results and Discussion}

The neutron diffraction experiment resulted in maps of two stress components and $\mathrm{d}_{0}$-values in the transverse cross-section of each sword which were determined with sub-millimeter spatial resolution. These two material characteristics are the imprint of the production process and their study can help to identify the actual manufacturing techniques based on qualitative and quantitative data rather than stylistic criteria.

Although 2D maps give a good idea about spatial distribution, in order to facilitate the interpretation of data only detailed 1D views of the central line are provided in Fig. 3. To make results geometrically comparable all curves are plotted in the scale normalized to 1 . The $2 \mathrm{D}$ maps omitted in this paper will be reported and more in-depth discussed in a separate publication.

Somewhat contrary to expectation no blade clearly showed a layered structure even with experimental spatial resolution of $0.5 \mathrm{~mm}$. Through thickness profiles of stress, $\mathrm{d}_{0}$ and peak width are all smooth functions (see Fig. $2 \mathrm{~b}$ as an example). Although features with smaller dimensions are not detectable due to the gauge volume averaging, the main laminae (differing in $\mathrm{C}$ content and microstructure) were expected to be detectable since their size, 1-2 $\mathrm{mm}$, is larger than the gauge volume used in the measurement (Fig. 2). The most evident features for all blades are associated with the cutting edge area. The peak width and $\mathrm{d}_{0}$-values peak at this area which is obviously attributed to the special thermo-mechanical treatment the edge receives in the production process with typical water quenching of the edge and formation of martensite. Since in the angle-dispersive neutron diffraction experiment with a single diffraction peak, Fe (211) in our case, there is no possibility to distinguish between ferrite and martensite, the presence of martensite is manifested as both peak broadening and rapid variation of $\mathrm{d}_{0}$. There are some more subtle variations of $\mathrm{d}_{0}$ and peak width (for example very small changes through thickness in blade H4839), that can be attributed to different degree of cementation and formation of various pearlitic/bainitic or even martensitic microstructures depending on carbon content. Since the process of carbon diffusion from different original pieces is complex, formation of gradients and variations of microstructures in a blade steel is not surprising in quenched areas. In principle, this should be reflected in the patterns of $\mathrm{d}_{0}$ and peak width through mechanism of microstress due to thermal, elastic and plastic mismatch between ferrite and cementite composing pearlite/bainite. However, in comparison with the effects of martensite, those effects are much smaller, e.g. $\mathrm{d}_{0}$ shift of $-0.0003 \AA$ due to typical microstress in ferrite of $-120 \mathrm{MPa}$ and almost no peak broadening in ferrite [8]. Therefore, the dominant features are due to the presence of martensite.

Yet another commonality for all blades is a very weak transverse stress component which has almost no changes along the central line, though some variation in through thickness is clearly present, most likely due to forging action leaving surface layers in slight relative compression in respect to the inner layers (the corresponding figure is omitted here to streamline the paper). The longitudinal stress component is dominant and stress state can be considered as almost onedimensional. Thereafter, only longitudinal component is considered, often referred to as simply "residual stress".

The martensitic pattern at the cutting edge can be considered as a distinctive feature of one manufacturing school and, in some cases, its unique profile was purposely created as a visual instantaneously recognizable signature of the author. Therefore the martensitic area can be more or less extended from blade to blade according to manufacturing tradition or blacksmith's intention. 
Therefore, the pattern of martensitic microstructure can be used as a distinct signature of the sword making school/author. Visual inspection of the patterns plotted in Fig. 3 suggests a similarity of the blades H5378 and H4839 (blue and green). They share almost identical pattern for the stress, $\mathrm{d}_{0}$ and peak width parameter. It cannot be said the same for the blades H1360 and H6856 (black and red in Fig. 3) whose $d_{0}$ don't match in any of the characteristics. However, the following considerations can indicate otherwise. It is known that some of old blades with long history of usage were subjected to refurbishment and renewal of the cutting edge and blade surfaces, especially if damaged. If we assume that blade H1360 over its lifetime lost some $3 \mathrm{~mm}$ of material due to repetitious polishing and/or restoration upon possible damage of the cutting edge, after dimensional re-normalization (so that the modern cutting edge actually corresponds to $3 \mathrm{~mm}$ into depth location of the original blade), the patterns demonstrate remarkable similarity (Fig. 4). In fact, the match of the stress patterns might have an even better match if corrections for force and moment balance are introduced, since material under compressive stress was probably removed by re-polishing therefore distorting the original stress distribution to some extent. Although this consideration cannot be considered as the final proof, the experimental observation cannot be disregarded as a mere speculation.

Comparing the two groups, the following differences are the most obvious and most significant. For the H5378/H4839 the effect of martensite presence is more pronounced resulting in a wider diffraction peak and much larger $\mathrm{d}_{0}$. Quenching, accompanied with the martensitic transformation, also results in formation of the compressive stress zone in the area of cutting edge, some first $5 \mathrm{~mm}$ starting from the cutting edge (Fig. 3). These compressive stresses (and stress momentum) are compensated by tensile stresses in the core of the blade. In the pair H1360/H6856 this happens in a way similar to a simple bending with a typical almost linear slope on the opposite side (Fig. 4). However, for the pair H5378/H4839, another compressive zone is created on the back side of the sword, which is more deeply extended into the interior, up to $10 \mathrm{~mm}$ from the back (Fig. 3).

Although this can be associated with quenching, (without martensite transformation, since there is no apparent change either in $\mathrm{d}_{0}$ or peak width) but it is most probably related to the use of soft steel at the back of the blades (e.g. Honsanmai style, Fig. 1) that acquired a compressive stress during deformation in combination with harder steel of the adjacent areas.

Based on these comparative data, the mumei blade H6856 might date back to the oldest of the historical periods (Koto age, 987-1596 A.D.), to which blade H1360 is certainly attributed. The period the blade is stylistically attributed (c. 1600 A.D.) is known as a time of transition when the Shogun extended his control also to the manufacture of weapons in the frame of its political strategy of unification (Shinto age, 1596-1781 A.D.). As a consequence, the differentiation among various traditions that were developed during the previous age slowly dissolved, only remaining as a sort of merely stylistic differentiation with no real connection to the working process [2].

\section{Summary}

For the first time a detailed strain mapping of the Japanese katana blades was achieved with $0.5 \mathrm{~mm}$ resolution using non-destructive neutron diffraction technique. Three quantities were analyzed, stress $\mathrm{d}_{0}$-value and peak width, and interpolated 2D maps were acquired making possible mutual comparison of the blades based on the signature patterns of these measured quantities.

The analysis of four blades revealed two groups of blades, each with a characteristic pattern of stress, $\mathrm{d}_{0}$ and peak width. While one blade H6856 (c. 1600) was of unknown origin, through its characteristic pattern association it was attributed to the tradition common with the oldest blade in the collection H1360 (Sadatsugu, 1346-1370).

This study lays down a foundation for a future analysis of the Japanese blades and proves feasibility of the approach with $0.5 \mathrm{~mm}$ spatial resolution mapping.

These results will provide the MAAS with new valuable information on these swords which will be available to researchers, museum collection managers, the general public and future generations. 

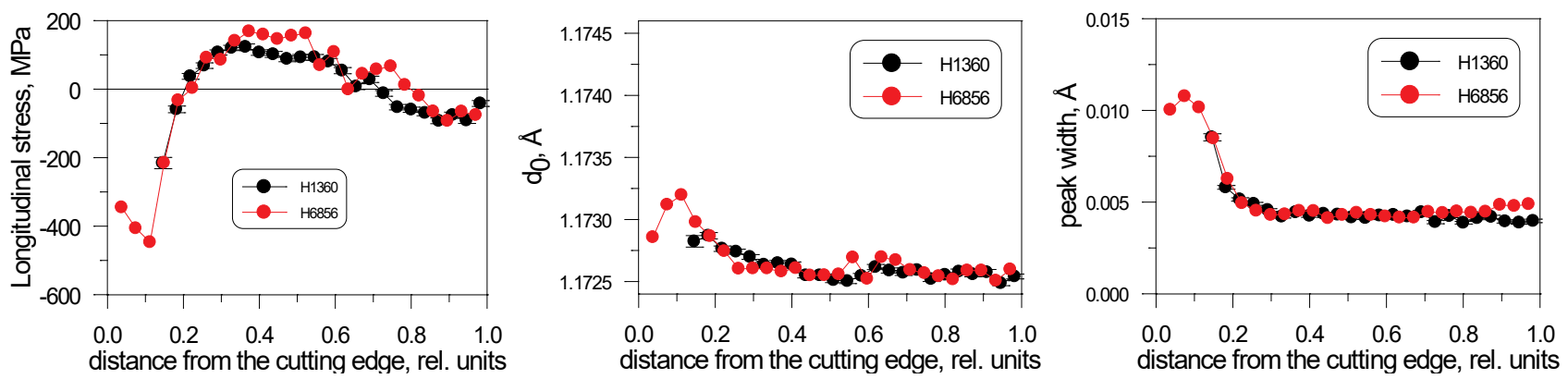

Fig. 4. Central line profiles of three material characteristics (stress, $d_{0}$ and peak width) for two blades, H1360 and H6856, with re-normalisation of the width of blade H1360.
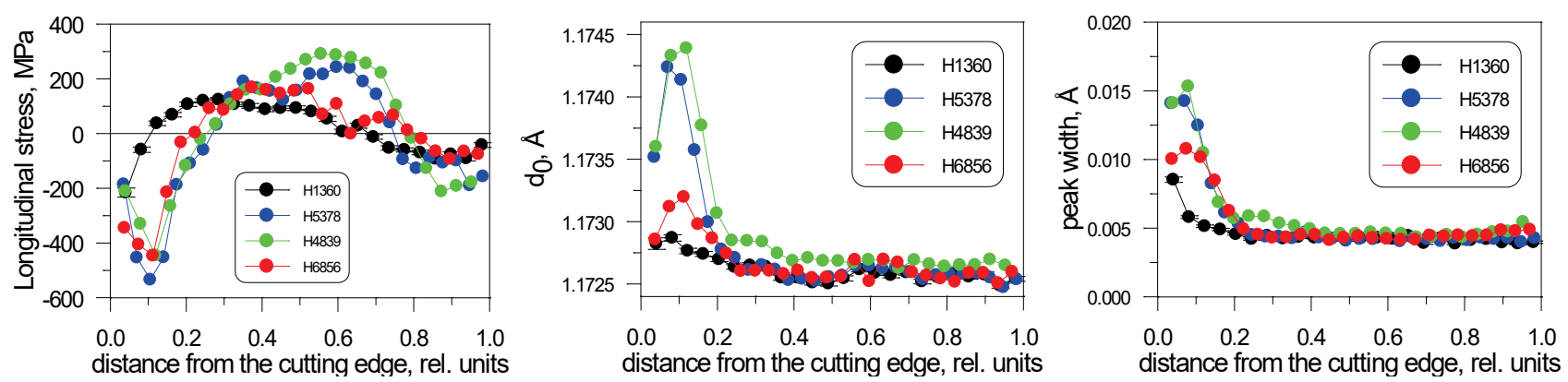

Fig. 3. Central line profiles of three material characteristics (stress, $d_{0}$ and peak width) for all blades.

\section{References}

[1] J.M. Yumoto, The Samurai Sword. A handbook. Tuttle, Tokyo (1958)

[2] K. Nagayama, The Connoisseurs Book of Japanese Swords, Kodansha International (1997)

[3] W.M. Hawley, Laminating Techniques in Japanese Swords (1974)

[4] F. Grazzi et al., From Koto age to modern times: Quantitative characterization of Japanese swords with Time of Flight Neutron Diffraction, Anal. At. Spectrom. 26 (5) (2011) 1030-1039. http://dx.doi.org/10.1039/c0ja00238k

[5] F. Salvemini et al., Neutron computed laminography on ancient metal artefacts, Anal. Methods 7 (2015) 271-278. http://dx.doi.org/10.1039/C4AY02014F

[6] O. Kirstein, V. Luzin, U. Garbe, The Strain-Scanning Diffractometer Kowari, Neutron News 20 (4) (2009) 34-36. http://dx.doi.org/10.1080/10448630903241175

[7] Ruiz-Hervias et al., Residual Stress Distributions in Bi-Metal (Ferritic to Austenitic Steel) Joints Made by Laser Welding, Mater. Sci. Forum. 772 (2013) 181-185 http://dx.doi.org/10.4028/www.scientific.net/MSF.772.181

[8] P. Van Houtte, K. Van Acker, J. Root, 1999, Residual Stress Determination in the Cementite and Ferrite Phases of High Carbon Steel, Textures and Microstructures 33 (1-4) (1999) 187-206. http://dx.doi.org/10.1155/TSM.33.187 